\title{
VORONOÏ-ALGORITHM EXPANSION OF TWO FAMILIES WITH PERIOD LENGTH GOING TO INFINITY
}

\author{
BRIGITTE ADAM
}

\begin{abstract}
We consider families of orders of complex cubic fields introduced recently by Levesque and Rhin and find the Voronoï-algorithm expansions and the fundamental units. We compare with the Jacobi-Perron algorithm expansions.
\end{abstract}

\section{INTRODUCTION}

A common problem of number theory is the search for parametrized families of positive integers $N$ such that the field $\mathbb{Q}(\sqrt{N})$ has a fundamental unit which is simply written according to the parameters. Such families have been given by Halter-Koch [5] and Williams [12]. In the complex cubic case, the fundamental unit of infinite families of fields $\mathbb{Q}(\sqrt[3]{M})$ is given by Stender [10]. For some of these families, the Voronoï-algorithm expansion [1], [2] and [11], which generalizes the continued fraction algorithm to three dimensions, has been calculated by Dubois [2] (with period length 1 or 2) and by Williams [12] (with period length less than or equal to 6). Levesque and Rhin [7] presented the Jacobi-Perron algorithm [9] expansion (another generalization of the continued fraction algorithm to higher dimensions) of two parametrized infinite families $\mathbb{Q}(\alpha)$, each depending on two parameters. These expansions being periodic (with the period length going to infinity), they obtained a unit of these fields and conjectured that this unit is fundamental in the order $\mathbf{Z}[\alpha]$. Fahrane [4] proved this for one of these families when one of the parameters is large enough (a noneffective result), whereas Louboutin [8] proved that this unit is a bounded power (the bound does not depend on the parameters) of the fundamental unit in the order $\mathbf{Z}[\alpha]$.

In this paper we provide a result which allows us to give the Voronoï-algorithm expansion of these two families. We obtain the following results :

- the period length of these expansions goes to infinity.

- the unit given by Levesque and Rhin is fundamental in the order $\mathbf{Z}[\alpha]$.

- for one of these families, Voronoï and Jacobi-Perron algorithms are the same, i.e., the Jacobi-Perron algorithm provides exactly all the minimal points given by the Voronoï algorithm.

Received by the editor November 29, 1993 and, in revised form, July 7, 1994.

1991 Mathematics Subject Classification. Primary 11Y65, 11R27, 11A55, 11R29.

(c)1995 American Mathematical Society 
Kühner [6] also presented the Voronoï-algorithm expansion of one of these families, and Dubois and Fahrane [3] study the second one.

\section{Minimal POINTS SEARCH METHOD}

Definition 2.1. Let $\alpha_{1}, \alpha_{2}$ be two real numbers so that $1, \alpha_{1}, \alpha_{2}$ are independent over the rationals. We let $L=\left\langle 1, \alpha_{1}, \alpha_{2}\right\rangle=\mathbf{Z}+\mathbf{Z} . \alpha_{1}+\mathbf{Z} . \alpha_{2}$ and for all $P=(u, v, w)$ (respectively $Q$ ) in $\mathbf{Z}^{3}$ we define $\psi=\psi(P)=u+v \alpha_{1}+w \alpha_{2}$ (respectively $\phi=\phi(Q)$ ). Let $F$ be a positive quadratic form with real coefficients of rank 2 so that $F(1,0,0)=1$ and $F(0,0,1)>1$. We say that $\psi$ is a minimal point adjacent to 1 on the right (further on, we will not specify "right") in relation to $L$ and $F$ if $\psi=\min \{\phi$ such that $\phi>1$ and $F(Q)<1\}$.

In this section we will give a proposition which, using an isotropic vector of the quadratic form, allows us to restrict to 5 the number of choices for a minimal point adjacent to 1 .

We will assume in the rest of this section that $\left(\omega_{2}, 1, \omega_{1}\right)$ is an isotropic vector of $F$, and we define

$$
\begin{array}{ll}
\phi_{1}=\left[\omega_{2}\right]+\alpha_{1}, & Q_{1}=\left(\left[\omega_{2}\right], 1,0\right), \\
\phi_{2}=\left[\omega_{2}\right]+\alpha_{1}+\alpha_{2}, & Q_{2}=\left(\left[\omega_{2}\right], 1,1\right), \\
\phi_{3}=\left[\omega_{2}\right]+\alpha_{1}-\alpha_{2}, & Q_{3}=\left(\left[\omega_{2}\right], 1,-1\right), \\
\phi_{4}=\left[\omega_{2}\right]-1+\alpha_{1}, & Q_{4}=\left(\left[\omega_{2}\right]-1,1,0\right), \\
\phi_{5}=\left[\omega_{2}\right]-1+\alpha_{1}+\alpha_{2}, & Q_{5}=\left(\left[\omega_{2}\right]-1,1,1\right), \\
\phi_{6}=\left[\omega_{2}\right]+1+2 \alpha_{1}-\alpha_{2}, & Q_{6}=\left(\left[\omega_{2}\right]+1,2,-1\right), \\
\phi_{7}=\left[\omega_{2}\right]+2 \alpha_{1}, & Q_{7}=\left(\left[\omega_{2}\right], 2,0\right), \\
\phi_{8}=\left[\omega_{2}\right]+1+\alpha_{1}-\alpha_{2}, & Q_{8}=\left(\left[\omega_{2}\right]+1,1,-1\right),
\end{array}
$$

where [...] is the greatest integer function. If $0<\alpha_{1}<1,0<\alpha_{2}<1$, we see that

$$
\left\{\begin{array}{l}
\phi_{4}<\phi_{3}<\phi_{1}<\phi_{2} \\
\phi_{4}<\phi_{5}<\phi_{1}<\phi_{2} \\
\phi_{1}<\phi_{7}<\phi_{6} \\
\phi_{1}<\phi_{8}<\phi_{6}
\end{array}\right.
$$

and

$$
\left\{\begin{array}{l}
\text { if } \alpha_{2}<\alpha_{1}, \text { then } \phi_{2}<\phi_{7}, \\
\text { if } 2 \alpha_{2}-1<\alpha_{1}<\alpha_{2}, \text { then } \phi_{7}<\phi_{2}<\phi_{6}, \\
\text { if } \alpha_{1}<2 \alpha_{2}-1, \text { then } \phi_{7}<\phi_{6}<\phi_{2}, \\
\text { if } 2 \alpha_{2}-1<0, \text { then } \phi_{2}<\phi_{8} .
\end{array}\right.
$$

Lemma 2.2. Let $F$ be a positive quadratic form in three variables with real coefficients of rank 2 such that

$$
F(1,0,0)=1 \text { and } F(0,0,1)>1 .
$$

Suppose that $F$ has an isotropic vector $\left(\omega_{2}, 1, \omega_{1}\right)$. Then we can write

$$
F(u, v, w)=a\left(w-\omega_{1} v\right)^{2}+2 b\left(w-\omega_{1} v\right)\left(u-\omega_{2} v\right)+\left(u-\omega_{2} v\right)^{2}
$$


and

$$
\begin{aligned}
F(u, v, w)= & \frac{a}{2}\left[w-\left(\omega_{1}+2 \frac{b}{a} \omega_{2}\right) v+2 \frac{b}{a} u\right]^{2}+\frac{a}{2}\left(w-\omega_{1} v\right)^{2} \\
& +\left(1-2 \frac{b^{2}}{a}\right)\left(u-\omega_{2} v\right)^{2}
\end{aligned}
$$

with $a>1$ and $b^{2}<a$.

Proof. Let $M$ be the matrix of the polar form associated with $F$. Writing

$$
M=\left(\begin{array}{ccc}
1 & a_{12} & a_{13} \\
a_{12} & a_{22} & a_{23} \\
a_{13} & a_{23} & a_{33}
\end{array}\right)
$$

with $a_{33}>1$, we deduce

$$
\left\{\begin{array}{l}
a_{12}=-\omega_{2}-\omega_{1} a_{13} \\
a_{22}=\left(\omega_{2}\right)^{2}+2 \omega_{1} \omega_{2} a_{13}+a_{33}\left(\omega_{1}\right)^{2} \\
a_{23}=-a_{13} \omega_{2}-a_{33} \omega_{1}
\end{array}\right.
$$

since $\left(\omega_{2}, 1, \omega_{1}\right)$ is an isotropic vector of $F$. If we write $a=a_{33}$ and $b=$ $a_{13}$, we obtain the formulas (1) and (2). Since $F$ is a positive form of rank 2 , we have $b^{2}<a$.

Now we can state the next proposition.

Proposition 2.3. If $0<\omega_{1}<1, \omega_{2}>1,0<\alpha_{1}<1,0<\alpha_{2}<1$ and $4 b^{2}<a$, we have

1. If $F\left(Q_{1}\right)<1$ :

a. if $b<0$, then the minimal point adjacent to 1 is $\phi_{1}, \phi_{3}$ or $\phi_{4}$;

b. if $b \geq 0$, then the minimal point adjacent to 1 is $\phi_{1}$ or $\phi_{5}$.

2. If $F\left(Q_{1}\right)>1$ and $F\left(Q_{2}\right)<1$ :

a. if $b<0$, then the minimal point adjacent to 1 is:

i. $\phi_{2}, \phi_{3}$ or $\phi_{4}$ if $\alpha_{2}<\alpha_{1}$,

ii. $\phi_{2}, \phi_{3}, \phi_{4}$ or $\phi_{7}$ if $2 \alpha_{2}-1<\alpha_{1}<\alpha_{2}$,

iii. $\phi_{2}, \phi_{3}, \phi_{4}, \phi_{6}$ or $\phi_{7}$ if $\alpha_{1}<2 \alpha_{2}-1$;

b. if $b \geq 0$, then the minimal point adjacent to 1 is:

i. $\phi_{2}$ or $\phi_{5}$ if $2 \alpha_{2}-1<0$,

ii. $\phi_{2}, \phi_{5}$ or $\phi_{8}$ if $2 \alpha_{2}-1>0$.

Remark. Inequality $|2 b|<1$ implies $4 b^{2}<a$ (since $a>1$ ).

Proof of Proposition 2.3. Let $\psi=u+v \alpha_{1}+w \alpha_{2}$ be the minimal point adjacent to 1 .

1. We assume first that $F\left(Q_{1}\right)<1$.

a. We first claim that $v \neq 0$. If $v=0$ we have :

if $u=0$, then $F(P)=a w^{2}>1$; if $w=0$, then $F(P)=u^{2} \geq 1$; and

if $u \neq 0$ and $w \neq 0$, then $F(P)>\frac{a}{2}+\left(1-2 \frac{b^{2}}{a}\right)>1$, which is impossible.

b. Next, we claim that if $\psi \neq \phi_{3}$, then $u, v, w$ are all nonnegative. 
Since $F(P)<1$ and $4 b^{2}<a$, we have $\left(u-\omega_{2} v\right)^{2}<2$; but $\omega_{2}>1$, then $u v \geq 0$. We have $\left(w-\omega_{1} v\right)^{2}<2$, then $w v \geq 0$ or $|w| \leq 1$. If $w v \geq 0$, then $v<0$ implies that $u \leq 0$ and $w \leq 0$, which is impossible because $\psi>0$, so we have $v>0, u \geq 0$ and $w \geq 0$. If $w v<0$, then $|w|=1$. If $w=1$, then $v<0$ and $u \leq 0$. If $u=0$, we have $F(P)>\frac{a}{2}+\left(1-2 \frac{b^{2}}{a}\right)>1$, and if $u<0$, we have $\psi<0$, which is impossible.

If $w=-1$, then $v>0, u \geq 0$ and $\left(w-\omega_{1} v\right)^{2}>1$, and if $u<\left[w_{2} v\right]$, then $\left(u-w_{2} v\right)^{2} \geq 1$ and $F(P)>1$; if $u=\left[\omega_{2} v\right]$, then $\psi=\phi_{3}$ or $\psi>\phi_{1}$; and if $u \geq\left[\omega_{2} v\right]+1$, then $\psi>\phi_{1}$.

Therefore, $w v<0$ implies that $\psi=\phi_{3}$.

Thus, we have proved that if $\psi \neq \phi_{3}$, then $v>0$, and $u$ and $w$ are nonnegative.

c. We claim that $v=1$.

For, if $v \geq 2$, we have $\left(u-\omega_{2} v\right)^{2}<2$, then $u>2\left[\omega_{2}\right]-\sqrt{2}$ and $u \geq\left[\omega_{2}\right]$, so $\psi>\phi_{1}$.

d. Study of $u$ and $w$.

Since $u \geq\left[\omega_{2}\right]-1$, we have $\left(u-\omega_{2} v\right)^{2}<2$.

We claim that $w<2$.

If $w \geq 2$ and $u \geq\left[\omega_{2}\right]$, then $\psi>\phi_{2}>\phi_{1}$; and if $u=\left[\omega_{2}\right]-1$, then $\left(u-\omega_{2} v\right)^{2}>1$ and $\left(w-\omega_{1} v\right)^{2}>1$, so $F(P)>1$.

If $u \geq\left[\omega_{2}\right]+1$, then $\psi>\phi_{2}>\phi_{1}$.

In case $w=1$, if $u=\left[\omega_{2} v\right]$, then $\psi>\phi_{1}$, so $u=\left[\omega_{2}\right]-1$ and $\psi=\phi_{5}$.

In case $w=0$, if $u=\left[\omega_{2} v\right]$, then $\psi=\phi_{1}$; and if $u=\left[\omega_{2}\right]-1$, then $\psi=\phi_{4}$.

Moreover, if $b<0$, we have $F\left(Q_{5}\right)>1$; and if $b \geq 0$, we have $F\left(Q_{3}\right)>1$ and $F\left(Q_{4}\right)>1$. Thus, the first part of the proposition is proved.

2. Let us assume now that $F\left(Q_{1}\right)>1$ and $F\left(Q_{2}\right)<1$.

As before, we have $u \geq 0, v>0$ and $w \geq 1$.

a. We assert that $v \leq 2$.

If $v \geq 3$, we have $u \geq\left[\omega_{2}\right]+1$; and if $w \geq 0$, then $\psi>\phi_{2}$. If $w=-1$ and $u>\left[\omega_{2}\right]+1$, then $\psi>\phi_{2}$; and if $u=\left[\omega_{2}\right]+1$, we have $\left(u-\omega_{2} v\right)^{2}>1$ and $\left(w-\omega_{1} v\right)^{2}>1$, so $F(P)>1$. Therefore, $v=1$ or $v=2$.

b. The case $v=1$. As in the proof of the first part, we have $u \geq$ $\left[\omega_{2}\right]-1$ and $w<2$.

In the case $w=1$, if $u>\left[\omega_{2}\right]$, then $\psi>\phi_{2}$; if $u=\left[\omega_{2}\right]$, then $\psi=\phi_{2} ;$ and if $u=\left[\omega_{2}\right]-1$, then $\psi=\phi_{5}$.

In the case $w=0$, if $u>\left[\omega_{2}\right]$, then $\psi>\phi_{2}$; if $u=\left[w_{2}\right]$, then $\psi=\phi_{1}$; and if $u=\left[\omega_{2}\right]-1$, then $\psi=\phi_{4}$.

In the case $w=-1$, if $u>\left[\omega_{2}\right]+1$, then $\psi>\phi_{2}$; if $u=\left[\omega_{2}\right]+1$, then $\psi=\phi_{8}$; if $u=\left[\omega_{2}\right]$, then $\psi=\phi_{3}$; and if $u=\left[\omega_{2}\right]-1$, we have $\left(w-\omega_{1} v\right)^{2}>1$ and $\left(u-\omega_{2} v\right)^{2}>1$, so $F(P)>1$.

c. The case $v=2$. In this case $u \geq\left[\omega_{2}\right]$.

If $w \geq 1$, then $\psi>\phi_{2}$. 
In the case $w=0$, if $u>\left[\omega_{2}\right]$, then $\psi>\phi_{2} ;$ and if $u=\left[\omega_{2}\right]$, then $\psi=\phi_{7}$.

In the case $w=-1$, if $u>\left[\omega_{2}\right]+1$, then $\psi>\phi_{2}$; if $u=\left[\omega_{2}\right]+1$, then $\psi=\phi_{6}$; and if $u=\left[\omega_{2}\right]$, then $\left(w-\omega_{1} v\right)^{2}>1$ and $(u-$ $\left.\omega_{2} v\right)^{2}>1$, so $F(P)>1$.

Moreover, if $b<0$ we have $F\left(Q_{5}\right)>1$ and $F\left(Q_{8}\right)>1$; and if $b \geq 0$, we have $F\left(Q_{3}\right)>1, F\left(Q_{4}\right)>1, F\left(Q_{7}\right)>1$ and $F\left(Q_{6}\right)>1$. Thus, the second part of the proposition is proved.

\section{VORONOÏ ALGORITHM}

Let $K$ be a cubic algebraic number field of negative discriminant and $L$ a lattice $\left(L \subseteq \mathbb{R}^{3}\right)$ of $K$ with basis $\left\{1, \alpha_{1}, \alpha_{2}\right\}$. As before, to each point $P=(u, v, w)$ (respectively $Q)$ in $\mathbf{Z}^{3}$ there corresponds an element $\psi=$ $\psi(P)=u+v \alpha_{1}+w \alpha_{2}$ (respectively $\left.\phi=\phi(Q)\right)$ in $L$, and we define

$$
F(P)=\frac{N(\psi)}{\psi}=\psi^{\prime} \psi^{\prime \prime},
$$

where $N$ denotes the norm of $K$ over $\mathbb{Q}$, and $\psi^{\prime}$ and $\psi^{\prime \prime}$ the conjugates of $\psi$.

Definition 3.1. We say that $\psi=\psi(P)$ is a minimal point of $L$ if for all $\phi=$ $\phi(Q)$ in $L$ so that $0<\phi<\psi$ we have $F(Q)>F(P)$. We define the increasing chain of the minimal points of $L$ by :

$$
\begin{gathered}
\psi_{0}=1, \\
\psi_{k+1}=\min \left\{\psi \text { such that } \psi>\psi_{k} \text { and } F(P)<F\left(P_{k}\right)\right\} \text { if } k \geq 0 .
\end{gathered}
$$

Then $\psi_{k+1}$ is the minimal point adjacent (on the right) to $\psi_{k}$ in $L$. Let $\mathscr{O}$ be any order of $K$ and $L=\mathscr{O}$. By Voronoï we know that the previous chain is of the purely periodic form :

$$
\ldots, \epsilon^{-1} \psi_{l-1}, 1, \psi_{1}, \ldots, \psi_{l-1}, \psi_{l}=\epsilon, \epsilon \psi_{1}, \ldots, \epsilon \psi_{l-1}, \ldots,
$$

where $l$ denotes the period length and $\epsilon$ is the fundamental unit of $\mathscr{O}$. To calculate such a sequence, it is sufficient to know how to construct the minimal point adjacent to 1 in a lattice $L=\left\langle 1, \alpha_{1}, \alpha_{2}\right\rangle$. Indeed, let $\psi_{0}=1$ and $\psi_{1}$ be the minimal point adjacent to 1 in $L_{0}=\mathscr{O}=\left\langle 1, \alpha_{1}, \alpha_{2}\right\rangle$.

a. We choose an auxiliary point $\phi_{1}$ so that $\left\{\psi_{1}, \phi_{1}, \psi_{0}\right\}$ is a basis of $L_{0}$.

b. $\psi_{2}$ is the minimal point adjacent to $\psi_{1}$ in $\mathscr{L}_{1}=\left\langle\psi_{1}, \phi_{1}, \psi_{0}\right\rangle$ is equivalent to $\frac{\psi_{2}}{\psi_{1}}$ being the minimal point adjacent to 1 in $L_{1}=\left\langle 1, \frac{\phi_{1}}{\psi_{1}}, \frac{\psi_{0}}{\psi_{1}}\right\rangle$.

This process can be continued by induction.

\section{Applications}

4.1. Study of the first family. Let $c \geq 2$ and $m \geq 1$ be two integers; we consider the polynomial

$$
f(X)=X^{3}-c^{m} X^{2}-(c-1) X-c^{m} .
$$

This case was considered by Fahrane [4] and by Kühner [6]. Levesque and Rhin [7] have shown that $f(X)$ is irreducible and has exactly one real root $\alpha$. 


\subsubsection{Statement of the theorem.}

Theorem 4.1. Let $\alpha$ be the real root of the polynomial $f(X), K=\mathbb{Q}(\alpha)$, and $\mathscr{O}=\mathrm{Z}[\alpha]$. Then

(i) The chain of the minimal points of $\mathscr{O}$ is: for $0 \leq s \leq m-1$

$$
\begin{gathered}
\psi_{0}=1, \quad \psi_{3 s+1}=\alpha\left(\frac{\alpha}{\alpha-c^{m}}\right)^{s}, \quad \psi_{3 s+2}=\alpha^{2}\left(\frac{\alpha}{\alpha-c^{m}}\right)^{s}, \\
\psi_{3 s+3}=\left(\frac{c \alpha}{\alpha-c^{m}}\right)^{s+1} \quad \text { and } \quad \psi_{3 m+1}=\alpha\left(\frac{\alpha}{\alpha-c^{m}}\right)^{m} .
\end{gathered}
$$

(ii) $\epsilon=\alpha\left(\frac{\alpha}{\alpha-c^{m}}\right)^{m}$ is the fundamental unit of $\mathscr{O}$ and the Voronoi-algorithm expansion period length is $l=3 m+1$.

4.1.2. Proof of Theorem 4.1. For this proof we need the following formulas :

$$
c^{m}<\alpha<c^{m}+\frac{c}{\alpha}
$$

and

$$
1+\frac{1}{\alpha^{2}}=\frac{c}{\alpha\left(\alpha-c^{m}\right)} .
$$

Let $L=\left\langle 1, \alpha_{1}, \alpha_{2}\right\rangle$ be a lattice in $K$ and $\psi$ the minimal point adjacent to 1 in $L$. Writing $\psi=u+v \alpha_{1}+w \alpha_{2}$, we have the following lemmas :

Lemma 4.2. For an integer $s, 0 \leq s \leq m$,

$$
\text { if } L=\left\langle 1, \alpha-c^{m}, \frac{c^{s}}{\alpha}\right\rangle \text {, then }(u, v, w)=\left(c^{m}, 1,0\right) .
$$

Proof. We verify in this case that $F$ is a positive quadratic form, which we can write in the form (1) and (2) with

$$
a=\frac{\alpha}{c^{m-2 s}}, \quad b=-\frac{\alpha\left(\alpha-c^{m}\right)}{2 c^{m-s}}, \quad \omega_{2}=\alpha, \quad \omega_{1}=\frac{c^{m-s}}{\alpha} .
$$

We have $0<\omega_{1}<1, \omega_{2}>1,0<\alpha_{1}<1,0<\alpha_{2}<1$ and $4 b^{2}<a$, since

$$
\frac{4 b^{2}}{a}=\frac{\alpha\left(\alpha-c^{m}\right)^{2}}{c^{m}}<1
$$

With the notation of $\S 2$, we have $\phi_{1}=\alpha$, so that

$$
F\left(Q_{1}\right)=\frac{N(\alpha)}{\alpha}=\frac{c^{m}}{\alpha}<1 \text { and } b<0 .
$$

According to Proposition 2.3, the minimal point adjacent to 1 is $\phi_{1}, \phi_{3}$ or $\phi_{4}$. But $Q_{3}=\left(c^{m}, 1,-1\right)$, and according to (2) we have

$$
F\left(Q_{3}\right)>\frac{\alpha}{2 c^{m-2 s}}\left(1+\frac{c^{m-s}}{\alpha}\right)^{2}>\frac{\alpha}{2 c^{m-2 s}}+c^{s}+\frac{c^{m}}{2 \alpha}>c^{s} \geq 1 .
$$

Finally, $\phi_{4}=\alpha-1$, and

$$
F\left(Q_{4}\right)=\frac{N(\alpha-1)}{\alpha-1}=\frac{2 c^{m}+c-2}{\alpha-1}>\frac{2 c^{m}+c-2}{c^{m}}>1 .
$$

Therefore, $\psi=\phi_{1}$, i.e., $(u, v, w)=\left(c^{m}, 1,0\right)$. 
Lemma 4.3. For an integer $s, 0 \leq s \leq m-1$,

$$
\text { if } L=\left\langle 1, \frac{c^{s}}{\alpha^{2}}, \frac{1}{\alpha}\right\rangle \text {, then }(u, v, w)=\left(c^{s}, 1,0\right) \text {. }
$$

Proof. As in the proof of Lemma 4.2, we have

$$
a=\frac{\alpha}{c^{m}}, \quad b=-\frac{\alpha\left(\alpha-c^{m}\right)}{2 c^{m}}, \quad \omega_{2}=\frac{c^{s} \alpha}{c^{m}}, \quad \omega_{1}=\frac{c^{s} \alpha}{c^{m}}\left(\alpha-c^{m}\right)
$$

and $0<\omega_{1}<1, \omega_{2}>1,0<\alpha_{1}<1,0<\alpha_{2}<1$. Moreover,

$$
|2 b|=\frac{\alpha\left(\alpha-c^{m}\right)}{c^{m}}<1 .
$$

Then we can use Proposition 2.3. We have

$$
\phi_{1}=\frac{c^{s+1}}{\alpha\left(\alpha-c^{m}\right)} \quad \text { and } \quad N\left(\alpha-c^{m}\right)=c^{m+1}
$$

so

$$
F\left(Q_{1}\right)=\frac{\alpha\left(\alpha-c^{m}\right)}{c^{2 m-2 s-1}}<1 \text { and } b<0 .
$$

We have $Q_{3}=\left(c^{s}, 1,-1\right)$, and from (2),

$$
F\left(Q_{3}\right)>\frac{\alpha}{2 c^{m}}\left[\left(1+c^{s}\left(\alpha-c^{m}\right)\right)^{2}+\left(1+\frac{c^{s} \alpha}{c^{m}}\left(\alpha-c^{m}\right)\right)^{2}\right]>1 .
$$

We have $Q_{4}=\left(c^{s}-1,1,0\right)$, and from (1),

$$
F\left(Q_{4}\right)=\frac{\alpha}{c^{m}}\left(\omega_{1}\right)^{2}+\frac{\alpha\left(\alpha-c^{m}\right)}{c^{m}} \omega_{1}\left(c^{s}-1-\frac{c^{s} \alpha}{c^{m}}\right)+\left(1+\frac{c^{s}\left(\alpha-c^{m}\right)}{c^{m}}\right)^{2} .
$$

Simplifying the two last terms, we obtain

$$
F\left(Q_{4}\right)=1+\frac{\alpha^{2}\left(\alpha-c^{m}\right)^{2}}{c^{2 m-s}}\left(c^{s}-1\right)+\frac{2 c^{s}\left(\alpha-c^{m}\right)}{c^{m}}+\frac{c^{2 s}}{c^{2 m}}\left(\alpha-c^{m}\right)^{2}>1 .
$$

Therefore, $\psi=\phi_{1}$, i.e., $(u, v, w)=\left(c^{s}, 1,0\right)$.

Lemma 4.4. For an integer $s, 0 \leq s \leq m-1$,

$$
\text { if } L=\left\langle 1, \frac{\alpha-c^{m}}{c^{s+1}}, \frac{\alpha\left(\alpha-c^{m}\right)}{c^{s+1}}\right\rangle \text {, then }(u, v, w)=\left(c^{m-1-s}, 1,0\right) \text {. }
$$

Proof. As before, we have

$$
a=\frac{c^{2 m-2 s-1}}{\alpha\left(\alpha-c^{m}\right)}, \quad b=\frac{c^{m-s-1}}{2}\left(\frac{c-1}{c^{m}}-\frac{1}{\alpha}\right), \quad \omega_{2}=\frac{c^{m-s}}{\alpha\left(\alpha-c^{m}\right)}, \quad \omega_{1}=\frac{1}{\alpha}
$$

and $0<\omega_{1}<1, \omega_{2}>1,0<\alpha_{1}<1,0<\alpha_{2}<1$. Moreover,

$$
|2 b|=c^{m-s-1}\left(\frac{c-1}{c^{m}}-\frac{1}{\alpha}\right)<c^{-s} \leq 1 \text {. }
$$

So we can use Proposition 2.3. We have

$$
\phi_{1}=\frac{\alpha}{c^{s+1}} \text { and } F\left(Q_{1}\right)=\frac{c^{m-2 s-2}}{\alpha}<1 \text { and } b>0,
$$

so $\psi=\phi_{1}$ or $\psi=\phi_{5}$. By using formula (1) for $F\left(Q_{5}\right)$ and $F\left(Q_{1}\right)$, we have

$$
F\left(Q_{5}\right)=F\left(Q_{1}\right)+1+\left(a-2 a \omega_{1}-2 b\right)+\left(2\left\{\omega_{2}\right\}-2 b\left\{\omega_{2}\right\}\right)+2 b \omega_{1},
$$


TABLE 1

\begin{tabular}{|c|c|c|c|}
\hline$k$ & $L_{k}=\left\langle 1, \frac{\phi_{k}}{\psi_{k}}, \frac{\psi_{k-1}}{\psi_{k}}\right\rangle$ & $\frac{\psi_{k+1}}{\psi_{k}}$ & $\frac{\phi_{k+1}}{\psi_{k}}$ \\
\hline 0 & $\left\langle 1, \alpha-c^{m}, \frac{c^{m}}{\alpha}\right\rangle$ & $\left(c^{m}, 1,0\right)$ & $(c-1,0,1)$ \\
\hline $3 s+1$ & $\left\langle 1, \alpha-c^{m}, \frac{c^{s}}{\alpha}\right\rangle$ & $\left(c^{m}, 1,0\right)$ & $(0,0,1)$ \\
\hline $3 s+2$ & $\left\langle 1, \frac{c^{s}}{\alpha^{2}}, \frac{1}{\alpha}\right\rangle$ & $\left(c^{s}, 1,0\right)$ & $(0,0,1)$ \\
\hline $3 s+3$ & $\left\langle 1, \frac{\alpha-c^{m}}{c^{s+1}}, \frac{\alpha\left(\alpha-c^{m}\right)}{c^{s+1}}\right\rangle$ & $\left(c^{m-1-s}, 1,0\right)$ & $(0,0,1)$ \\
\hline
\end{tabular}

where $\left\{\omega_{2}\right\}=\omega_{2}-\left[\omega_{2}\right]$. We claim that $a-2 a \omega_{1}-2 b>0$. Indeed,

$$
\frac{b}{c^{m-s-1}}<\frac{1}{2 c^{m-1}} \text {, hence } \frac{a-2 a \omega_{1}-2 b}{c^{m-s-1}}>\frac{c^{m-s}}{\alpha\left(\alpha-c^{m}\right)}\left(1-\frac{2}{\alpha}\right)-\frac{1}{c^{m-1}} \text {. }
$$

Since $\frac{c}{\alpha\left(\alpha-c^{m}\right)}=1+\frac{1}{\alpha^{2}}$, we have

$$
\frac{a-2 a \omega_{1}-2 b}{c^{m-s-1}}>c^{m-s-1}-2 \frac{c^{m-s-1}}{\alpha}+\frac{c^{m-s-1}}{\alpha^{2}}\left(1-\frac{2}{\alpha}\right)-\frac{1}{c^{m-1}} .
$$

If $s<m-1, c^{m-1-s} \geq 2,2 \frac{c^{m-s-1}}{\alpha}<1$ and $\frac{1}{c^{m-1}} \leq 1$ so $a-2 a \omega_{1}-2 b>0$, as claimed. If $s=m-1$, then

$$
a-2 a \omega_{1}-2 b=\left(1+\frac{1}{c^{m-1}}\right)+\left(\frac{1}{\alpha^{2}}-\frac{2}{\alpha^{3}}\right)+\left(\frac{1}{c^{m}}-\frac{1}{\alpha}\right)>0 .
$$

Moreover, $2\left\{\omega_{2}\right\}-2 b\left\{\omega_{2}\right\}>0$ so $F\left(Q_{5}\right)>1$. Therefore $\psi=\phi_{1}$, i.e., $(u, v, w)=\left(c^{m-1-s}, 1,0\right)$.

We prove the theorem by induction with the help of these lemmas in the following way.

Let $L_{0}=\left\langle 1, \alpha-c^{m}, \frac{c^{m}}{\alpha}\right\rangle$. According to Lemma 4.2 we have $\psi_{1}=\alpha$.

a. We choose $\phi_{1}=\alpha\left(\alpha-c^{m}\right)$.

b. We obtain $L_{1}=\left\langle 1, \alpha-c^{m}, \frac{1}{\alpha}\right\rangle$, and by the same lemma we have $\frac{\psi_{2}}{\psi_{1}}=\alpha$, i.e., $\psi_{2}=\alpha^{2}$.

If we continue this process, we obtain, for $0 \leq s \leq m-1$, the results given in Table 1. In the table we have written

$$
\phi_{0}=\alpha-c^{m}, \quad \psi_{-1}=\frac{c^{m}}{\alpha}
$$


and the third and fourth columns correspond to the coordinates of $\frac{\psi_{k+1}}{\psi_{k}}$ and of $\frac{\phi_{k+1}}{\psi_{k}}$ in the lattice $L_{k}$.

Now, the chain of minimal points $\psi_{k}$ of $\mathbf{Z}[\alpha]$ can easily be found with the help of the successive quotients $\frac{\psi_{k+1}}{\psi_{k}}$. Hence,

$$
\psi_{3 m+1}=\alpha\left(\frac{\alpha}{\alpha-c^{m}}\right)^{m} .
$$

We have

$$
N\left(\psi_{3 m+1}\right)=1 \text { and } N\left(\psi_{i}\right) \neq 1 \quad \text { if } 0<i \leq 3 m .
$$

Therefore, $\psi_{3 m+1}$ is the fundamental unit $\epsilon$ in $\mathscr{O}$, and the Voronoï-algorithm expansion period length is $l=3 m+1$.

\subsubsection{The Jacobi-Perron algorithm.}

Definition 4.5. Let $\alpha_{1}, \alpha_{2}$ be two real numbers. The Jacobi-Perron algorithm expansion of $\left(\alpha_{1}, \alpha_{2}\right)$ is given by two sequences $\left(a_{i}\right)\left(b_{i}\right),(i \geq 0)$ of integers defined by

$$
\left\{\begin{array}{l}
\alpha_{1}^{0}=\alpha_{1}, \quad \alpha_{2}^{0}=\alpha_{2} \\
\text { and for } \nu \geq 0: \quad a_{\nu}=\left[\alpha_{2}^{\nu}\right], b_{\nu}=\left[\alpha_{1}^{\nu}\right] \\
\alpha_{2}^{\nu+1}=\frac{1}{\alpha_{1}^{\nu}-b_{\nu}}, \quad \alpha_{1}^{\nu+1}=\frac{\alpha_{2}^{\nu}-a_{\nu}}{\alpha_{1}^{\nu}-b_{\nu}} .
\end{array}\right.
$$

Remark. The basis of the lattices $L_{k}, 0 \leq k \leq 3 m$, are given by the JacobiPerron algorithm expansion of $\left(\alpha\left(\alpha-c^{m}\right), \alpha\right)$.

For $0 \leq k \leq 3 m$ we define the transition matrix from $L_{k}$ to $L_{k+1}$ by

$$
M_{k}\left(\begin{array}{c}
\psi_{k} \\
\phi_{k} \\
\psi_{k-1}
\end{array}\right)=\left(\begin{array}{c}
\psi_{k+1} \\
\phi_{k+1} \\
\psi_{k}
\end{array}\right)
$$

The matrices $M_{k}$ are given by the previous lemmas, i.e.:

$$
M_{0}=\left(\begin{array}{ccc}
c^{m} & 1 & 0 \\
c-1 & 0 & 1 \\
1 & 0 & 0
\end{array}\right)
$$

and for $0 \leq s \leq m-1$,

$$
M_{3 s+1}=\left(\begin{array}{ccc}
c^{m} & 1 & 0 \\
0 & 0 & 1 \\
1 & 0 & 0
\end{array}\right), M_{3 s+2}=\left(\begin{array}{ccc}
c^{s} & 1 & 0 \\
0 & 0 & 1 \\
1 & 0 & 0
\end{array}\right), M_{3 s+3}=\left(\begin{array}{ccc}
c^{m-s-1} & 1 & 0 \\
0 & 0 & 1 \\
1 & 0 & 0
\end{array}\right) .
$$

According to Levesque and Rhin [7] we can write

$$
M_{k}=\left(\begin{array}{ccc}
a_{l-k} & 1 & 0 \\
b_{l-k} & 0 & 1 \\
1 & 0 & 0
\end{array}\right)
$$

where $l=3 m+1$ and $a_{l-k}, b_{l-k}$ are defined by the Jacobi-Perron algorithm expansion of $\left(\alpha\left(\alpha-c^{m}\right), \alpha\right)$. 
Remark. For the quadratic form $F$, an isotropic vector in $L_{k}$ has for $1 \leq k \leq$ $3 m$ the coordinates

$$
\left(\begin{array}{c}
\alpha_{2}^{k-1} \\
1 \\
\alpha_{1}^{k-1}-b_{k-1}
\end{array}\right),
$$

where $\alpha_{2}^{k-1}$ and $\alpha_{1}^{k-1}$ are defined by the Jacobi-Perron algorithm expansion of $\left(\alpha\left(\alpha-c^{m}\right), \alpha\right)$.

4.2. Study of the second family. Let $c \geq 2$ and $m \geq 1$ be two integers; we consider the polynomial

$$
f(X)=X^{3}-\left(c^{m}+c-1\right) X^{2}-\left(c^{m}-1\right) X-c^{m} .
$$

Levesque and Rhin [7] have shown that $f(X)$ is irreducible and has exactly one real root.

\subsubsection{Statement of the theorem.}

Theorem 4.6. Let $\alpha$ be the real root of the polynomial $f(X), K=\mathbb{Q}(\alpha)$, and $\mathscr{O}=\mathbf{Z}[\alpha]$. Then

(i) The chain of the minimal points of $\mathscr{O}$ is

$$
\begin{gathered}
\psi_{0}=1, \quad \psi_{1}=\alpha, \quad \psi_{2}=\alpha^{2}, \quad \psi_{3}=\frac{c \alpha^{2}}{\alpha-c^{m}} ; \\
\psi_{4 t}=\alpha\left(\frac{\alpha^{2}}{\alpha-c^{m}}\right)^{t}, \quad \psi_{4 t+1}=\alpha^{2}\left(\frac{\alpha^{2}}{\alpha-c^{m}}\right)^{t} \quad \text { for } 1 \leq t \leq m-1 ; \\
\psi_{4 t+2}=\frac{\alpha\left(c^{t+1}-1\right)+c^{m}}{\alpha-c^{m}} \alpha\left(\frac{\alpha^{2}}{\alpha-c^{m}}\right)^{t}, \quad \psi_{4 t+3}=\left(\frac{c \alpha^{2}}{\alpha-c^{m}}\right)^{t+1} \text { for } 1 \leq t \leq m-2 ; \\
\text { and } \psi_{4 m-2}=\left(\frac{c \alpha^{2}}{\alpha-c^{m}}\right)^{m}, \quad \psi_{4 m-1}=\alpha\left(\frac{\alpha^{2}}{\alpha-c^{m}}\right)^{m} ;
\end{gathered}
$$

(ii) The fundamental unit of $\mathscr{O}$ is $\epsilon=\alpha\left(\frac{\alpha^{2}}{\alpha-c^{m}}\right)^{m}$, and the Voronoi-algorithm expansion period length is $l=4 m-1$.

4.2.2. Proof of Theorem 4.6. For this proof we need the following formulas:

$$
c_{2}<\alpha<c_{2}+\frac{c^{m}}{\alpha}
$$

and

$$
1+\frac{1}{\alpha}+\frac{1}{\alpha^{2}}=\frac{c}{\alpha-c^{m}},
$$

where $c_{2}=c^{m}+c-1$. With the same notation as before we have the following lemmas :

Lemma 4.7. For an integer $t, 0 \leq t \leq m$,

$$
\text { if } L=\left\langle 1, \alpha-c_{2}, \frac{c^{t}}{\alpha}\right\rangle \text {, then }(u, v, w)=\left(c_{2}, 1,0\right) \text {. }
$$

Proof. The proof of this lemma is analogous to the one of Lemma 4.2 of the previous section. 
Lemma 4.8. For an integer $t, 0<t<m-1$,

$$
\text { if } L=\left\langle 1, \frac{c^{t}-1}{\alpha}+\frac{c^{t}}{\alpha^{2}}, \frac{1}{\alpha}\right\rangle, \text { then }(u, v, w)=\left(c^{t}, 1,0\right) \text {; }
$$

if $t=0$ or $t=m-1$,

$$
\text { if } L=\left\langle 1, \frac{c^{t}-1}{\alpha}+\frac{c^{t}}{\alpha^{2}}, \frac{1}{\alpha}\right\rangle, \text { then }(u, v, w)=\left(c^{t}, 1,1\right) \text {. }
$$

Proof. The coefficients $a$ and $b$ of the quadratic form $F$ in relation to $L$ and the isotropic vector are given by

$$
a=\frac{\alpha}{c^{m}}, \quad b=-\frac{\alpha\left(\alpha-c_{2}\right)}{2 c^{m}}, \quad \omega_{2}=\frac{\alpha}{c^{m-t}}, \quad \omega_{1}=\frac{\alpha\left(c^{m-t}-1\right)+c^{m}}{c^{m-t} \alpha}
$$

and $0<\omega_{1}<1, \omega_{2}>1,0<\alpha_{1}<1,0<\alpha_{2}<1$. Moreover,

$$
|2 b|=\frac{\alpha\left(\alpha-c_{2}\right)}{c^{m}}<1
$$

According to (1), we have

$$
\begin{aligned}
F\left(Q_{1}\right)= & \frac{\alpha}{c^{m}}\left[1-\frac{c^{t}}{c^{m} \alpha}\left(\alpha-c^{m}\right)\right]^{2}+c^{2 t}\left(\frac{\alpha}{c^{m}}-1\right)^{2} \\
& -\frac{\alpha\left(\alpha-c_{2}\right)}{c^{m}}\left[1-\frac{c^{t}}{c^{m} \alpha}\left(\alpha-c^{m}\right)\right] \frac{c^{t}}{c^{m}}\left(\alpha-c^{m}\right),
\end{aligned}
$$

so that, on expansion,

$$
\begin{aligned}
F\left(Q_{1}\right)= & \frac{\alpha}{c^{m}}+\frac{c^{2 t}}{c^{2 m}}\left(\alpha-c^{m}\right)^{2}\left(\frac{1}{c^{m} \alpha}+1+\frac{\alpha-c_{2}}{c^{m}}\right) \\
& -2 \frac{c^{t}}{c^{2 m}}\left(\alpha-c^{m}\right)-\frac{c^{t}}{c^{2 m}}\left(\alpha-c^{m}\right) \alpha\left(\alpha-c_{2}\right)
\end{aligned}
$$

We observe that

$$
\frac{1}{c^{m} \alpha}+1+\frac{\alpha-c_{2}}{c^{m}}=\frac{c}{\alpha-c^{m}}
$$

then

$$
F\left(Q_{1}\right)=\frac{\alpha}{c^{m}}-\frac{c^{t}}{c^{2 m}}\left(\alpha-c^{m}\right)\left[2+\alpha\left(\alpha-c_{2}\right)-c^{t+1}\right] .
$$

Thus, $F\left(Q_{1}\right)<1$ is equivalent to

$$
1-\frac{\alpha}{c^{m}}+\frac{c^{t}}{c^{2 m}}\left(\alpha-c^{m}\right)\left[2+\alpha\left(\alpha-c_{2}\right)-c^{t+1}\right]>0 .
$$

Multiplying by $\frac{c^{m}}{\alpha-c^{m}}$, and replacing $\alpha\left(\alpha-c_{2}\right)$ with $c^{m}-1+\frac{c^{m}}{\alpha}$, we see that this condition is equivalent to $1+c^{m}+\frac{c^{m}}{\alpha}-c^{t+1}-c^{m-t}>0$. Hence:

(i) if $0<t<m-1$, then $F\left(Q_{1}\right)<1$; 
(ii) if $t=0$ or $t=m-1$, then $F\left(Q_{1}\right)>1$, but in this case $\phi_{2}=\frac{c^{t+1}}{\alpha-c^{m}}$ and $N\left(\phi_{2}\right)=c^{2 m+1}$, so $F\left(Q_{2}\right)=\frac{\alpha-c^{m}}{c^{2 m-2 t-1}}<1$.

We have $F\left(Q_{3}\right)=F\left(Q_{1}\right)+a+2 a \omega_{1}+2 b \omega_{2} ;$ then

$$
F\left(Q_{3}\right)=F\left(Q_{1}\right)+\frac{\alpha}{c^{m}}+2 \frac{\alpha}{c^{m}} \frac{\alpha\left(c^{m-t}-1\right)+c^{m}}{c^{m-t} \alpha}-\frac{\alpha\left(\alpha-c_{2}\right)}{c^{m}} \frac{\alpha-c^{m}}{c^{m-t}} .
$$

We observe that $\alpha-c_{2}<2$ and that $\alpha-c^{m}<\frac{\alpha\left(c^{m-t}-1\right)+c^{m}}{\alpha}$ is equivalent to $c-1+\frac{c^{m}-1}{\alpha}+\frac{c^{m}}{\alpha^{2}}<c^{m-t}-1+\frac{c^{m}}{\alpha}$, i.e., $0<\left(c^{m-t}-c\right)+\frac{1}{\alpha}\left(c^{m}+1-\frac{c^{m}}{\alpha}\right)$, which is true. So $F\left(Q_{3}\right)>\frac{\alpha}{c^{m}}>1$.

We have

$$
F\left(Q_{4}\right)=F\left(Q_{1}\right)+2 b \omega_{1}+1+2\left\{\omega_{2}\right\}>F\left(Q_{1}\right)+2\left\{\omega_{2}\right\}
$$

so $F\left(Q_{4}\right)>F\left(Q_{1}\right)+2\left\{\omega_{2}\right\}$ since $-1<2 b \omega_{1}<0$; then

$$
F\left(Q_{4}\right)>\frac{\alpha}{c^{m}}-\frac{c^{t}}{c^{2 m}}\left(\alpha-c^{m}\right)\left[2+\alpha\left(\alpha-c_{2}\right)-c^{t+1}\right]+2 \frac{\alpha-c^{m}}{c^{m-t}} .
$$

The right-hand term is greater than 1 if and only if

$$
\frac{\alpha-c^{m}}{c^{m}}\left[1-\frac{c^{t}}{c^{m}}\left(2+\alpha\left(\alpha-c_{2}\right)-c^{t+1}\right)+2 c^{t}\right]>0
$$

which is equivalent ( replacing $\alpha\left(\alpha-c_{2}\right)$ with $\left.c^{m}-1+\frac{c^{m}}{\alpha}\right)$ to

$$
1+c^{t}+c^{2 t+1-m}-c^{t-m}-\frac{c^{t}}{\alpha}>0
$$

which is true for $0 \leq t \leq m-1$, so $F\left(Q_{4}\right)>1$. We use Proposition 2.3, observing that $b<0$.

(i) If $0<t<m-1$, then $\psi=\phi_{1}$, i.e., $(u, v, w)=\left(c^{t}, 1,0\right)$.

(ii) If $t=0$, then $2 \alpha_{2}-1<\alpha_{1}<\alpha_{2}$, so $\psi=\phi_{2}$ or $\phi_{7}$. Furthermore, $F\left(Q_{7}\right)=4 a \omega_{1}^{2}+8 b \omega_{1} \omega_{2}-4 b \omega_{1}\left[\omega_{2}\right]+\left(2 \omega_{2}-\left[\omega_{2}\right]\right)^{2}$ and $4 a \omega_{1}^{2}+8 b \omega_{1} \omega_{2}=0$ if $t=0$, so $F\left(Q_{7}\right)>1$ and $\psi=\phi_{2}$, i.e., $(u, v, w)=\left(c^{t}, 1,1\right)$. If $t=m-1$ and $m \geq 2$, then $\alpha_{1}>\alpha_{2}$, so $\psi=\phi_{2}$, i.e., $(u, v, w)=\left(c^{t}, 1,1\right)$.

Lemma 4.9. For an integer $t, 1 \leq t \leq m-2$,

$$
\text { if } L=\left\langle 1, \frac{\alpha-c^{m}}{\alpha\left(c^{t+1}-1\right)+c^{m}}, \frac{\alpha\left(\alpha-c^{m}\right)}{\alpha\left(c^{t+1}-1\right)+c^{m}}\right\rangle \text {, then }(u, v, w)=(1,1,0) \text {. }
$$


Proof. We have

$$
\begin{gathered}
a=\frac{c^{2 m+1}}{\left(\alpha-c^{m}\right)\left[\left(c^{t+1}-1\right)^{2}-\alpha\left(\alpha-c_{2}\right)\left(c^{t+1}-1\right)+c^{m} \alpha\right]}, \\
b=\frac{\left(c^{t+1}-1\right)\left(c_{2}-\alpha-2 c^{m}\right)+\alpha\left(\alpha-c_{2}\right)^{2}-2 c^{m}+c^{m} \alpha\left(\alpha-c_{2}\right)}{2\left[\left(c^{t+1}-1\right)^{2}-\alpha\left(\alpha-c_{2}\right)\left(c^{t+1}-1\right)+c^{m} \alpha\right]}, \\
\omega_{2}=\frac{c^{m-t} \alpha}{\alpha\left(c^{m-t}-1\right)+c^{m}}, \quad \omega_{1}=\frac{\alpha\left(\alpha-c^{m}\right)}{\alpha\left(c^{m-t}-1\right)+c^{m}},
\end{gathered}
$$

and $0<\omega_{1}<1, \omega_{2}>1,0<\alpha_{1}<1,0<\alpha_{2}<1$. Study of $b$ : writing $2 b=\frac{N}{D}$, we have

$$
N=\alpha\left(\alpha-c_{2}\right)^{2}+c^{m} \alpha\left(\alpha-c_{2}\right)-2 c^{m+t+1}-\left(\alpha-c_{2}\right)\left(c^{t+1}-1\right),
$$

hence

$$
N \geq \alpha\left(\alpha-c_{2}\right)^{2}+c^{m} \alpha\left(\alpha-c_{2}\right)-c^{2 m}-\left(\alpha-c_{2}\right)\left(c^{m-1}-1\right)=n
$$

we have

$$
n=\left(\alpha-c_{2}\right)\left[\alpha\left(\alpha-c_{2}\right)+c^{m} \alpha-\left(c^{m-1}-1\right)\right]-c^{2 m},
$$

so

$$
n=c^{m-1}\left(\alpha-c_{2}\right)\left[c-1+c \alpha+\frac{c}{\alpha}\right]-c^{2 m}
$$

replacing $\alpha\left(\alpha-c_{2}\right)$ with $c^{m}-1+\frac{c^{m}}{\alpha}$. Further,

$$
c^{1-m} n=\left(\alpha-c_{2}\right)(c-1)-\frac{c^{2}-c}{\alpha}=(c-1)\left(\alpha-c_{2} \frac{c}{\alpha}\right)>0 .
$$

We have $D>0$, so $b>0$. We claim that $|2 b|<1:$ this is equivalent to $N-D<0$. We have

$$
\begin{aligned}
N-D= & {\left[\alpha\left(\alpha-c_{2}\right)^{2}-c^{m+t+1}\right]+\left[c^{m} \alpha\left(\alpha-c_{2}\right)-c^{m} \alpha\right] } \\
& +\left[\alpha\left(\alpha-c_{2}\right)\left(c^{t+1}-1\right)-c^{m+t+1}\right]-\left(c^{t+1}-1\right)^{2}-\left(\alpha-c_{2}\right)\left(c^{t+1}-1\right) .
\end{aligned}
$$

So $N-D<0$, and $|2 b|<1$.

We have

$$
F\left(Q_{1}\right)=\frac{N\left(\phi_{1}\right)}{\phi_{1}}=\frac{c^{2 t+2}}{\left(c^{t+1}-1\right)^{2}-\alpha\left(\alpha-c_{2}\right)\left(c^{t+1}-1\right)+c^{m} \alpha}<1 ;
$$

therefore the minimal point adjacent to 1 is $\phi_{1}$ or $\phi_{5}$; but $\phi_{5}<1$, so $\psi=\phi_{1}$ i.e., $(u, v, w)=(1,1,0)$.

Lemma 4.10. For an integer $t, 1 \leq t \leq m-1$,

$$
\text { if } L=\left\langle 1, \frac{\alpha-c^{m}}{c^{t+1}}, \frac{\alpha\left(c^{t+1}-1\right)+c^{m}}{c^{t+1} \alpha}\right\rangle \text {, then }(u, v, w)=\left(c^{m-1-t}, 1,0\right) \text {. }
$$


Proof. We have

$$
\begin{array}{cc}
a=\frac{\left(c^{t+1}-1\right)^{2}-\alpha\left(\alpha-c_{2}\right)\left(c^{t+1}-1\right)+c^{m} \alpha}{c^{2 t+2}}, \quad b=\frac{2\left(c^{t+1}-1\right)-\alpha\left(\alpha-c_{2}\right)}{2 c^{t+1}}, \\
\omega_{2}=\frac{\alpha\left(c^{m-t}-1\right)+c^{m}}{\alpha\left(\alpha-c^{m}\right)}, \quad \omega_{1}=\frac{1}{\alpha}
\end{array}
$$

and $0<\omega_{1}<1, \omega_{2}>1,0<\alpha_{1}<1,0<\alpha_{2}<1$. Moreover, $4 b^{2}<a$. Indeed,

$$
a-4 b^{2}=\frac{1}{c^{2 t+2}}\left\{c^{m} \alpha-\alpha^{2}\left(\alpha-c_{2}\right)^{2}+3\left(c^{t+1}-1\right)\left[\alpha\left(\alpha-c_{2}\right)-\left(c^{t+1}-1\right)\right]\right\}>0 .
$$

We have

$$
F\left(Q_{1}\right)=\frac{c^{m}}{c^{2 t+2} \alpha}<1
$$

(i) If $t \leq m-2$, then $b<0$ and $\psi=\phi_{1}, \phi_{3}$ or $\phi_{4}$. But

$$
F\left(Q_{3}\right)>\frac{a}{2}\left(1+\frac{1}{\alpha}\right)^{2}>\frac{a}{2},
$$

and according to the inequalities $\left(c^{t+1}-1\right)^{2}>0, \alpha\left(\alpha-c_{2}\right)<c^{m},\left(c^{t+1}-1\right) \leq$ $\left(c^{m-1}-1\right)$ and $\alpha>c_{2}$, we obtain $a>2$, so $F\left(Q_{3}\right)>1$. According to (5) we have

$$
F\left(Q_{4}\right)=F\left(Q_{1}\right)+2 b \omega_{1}+1+2\left\{\omega_{2}\right\}>F\left(Q_{1}\right)+2\left\{\omega_{2}\right\} .
$$

To prove that $F\left(Q_{4}\right)>1$, it is sufficient to prove that $2 b \omega_{1}+1+2\left\{\omega_{2}\right\} \geq 0$. We have

$$
\begin{aligned}
2 b \omega_{1}+1+2\left\{\omega_{2}\right\}= & \frac{2\left(c^{t+1}-1\right)-\alpha\left(\alpha-c_{2}\right)}{c^{t+1} \alpha}+2\left(\frac{\alpha\left(c^{m-t}-1\right)+c^{m}}{\alpha\left(\alpha-c^{m}\right)}-c^{m-t-1}\right) \\
= & \frac{2}{c^{t+1}}\left[-\frac{1}{\alpha}+\frac{c^{m+1}}{\alpha-c^{m}}-c^{m}-\left(\alpha-c_{2}\right)\right] \\
& +\frac{\alpha-c_{2}}{c^{t+1}}+2\left[\frac{1}{\alpha}-\frac{1}{\alpha-c^{m}}+\frac{c^{m}}{\alpha\left(\alpha-c^{m}\right)}\right]
\end{aligned}
$$

and according to (4) the first term equals zero and so $F\left(Q_{4}\right)>1+\frac{\alpha-c_{2}}{c^{t+1}}>1$.

Therefore, $\psi=\phi_{1}$, i.e., $(u, v, w)=\left(c^{m-1-t}, 1,0\right)$.

(ii) If $t=m-1$, then $b>0$ and $\psi=\phi_{1}$ or $\phi_{5}$. We have

$$
F\left(Q_{5}\right)=\frac{\alpha(\alpha-1)+c^{m}}{c^{m} \alpha},
$$

and by multiplying the conjugates, we obtain

$$
F\left(Q_{5}\right)=\frac{\alpha\left(\alpha-c_{2}\right)+\alpha^{2}\left(\alpha-c_{2}\right)^{2}+c^{m}\left(\alpha^{2}-\alpha\right)+2 c^{m}}{c^{2 m} \alpha}>\frac{\alpha^{2}-\alpha}{c^{m} \alpha}>1 ;
$$

therefore, $F\left(Q_{5}\right)>1$ and $\psi=\phi_{1}$, i.e., $(u, v, w)=\left(c^{m-1-t}, 1,0\right)$.

We obtain for $1 \leq t \leq m-2$ the results given in Table 2 . 
TABLE 2

\begin{tabular}{|c|c|c|c|}
\hline$k$ & $L_{k}=\left\langle 1, \frac{\phi_{k}}{\psi_{k}}, \frac{\psi_{k-1}}{\psi_{k}}\right\rangle$ & $\frac{\psi_{k+1}}{\psi_{k}}$ & $\frac{\phi_{k+1}}{\psi_{k}}$ \\
\hline 0 & $\left\langle 1, \alpha-c_{2}, \frac{c^{m}}{\alpha}\right\rangle$ & $\left(c_{2}, 1,0\right)$ & $\left(c^{m}-1,0,1\right)$ \\
\hline 1 & $\left\langle 1, \alpha-c_{2}, \frac{1}{\alpha}\right\rangle$ & $\left(c_{2}, 1,0\right)$ & $(0,0,1)$ \\
\hline 2 & $\left\langle 1, \frac{1}{\alpha^{2}}, \frac{1}{\alpha}\right\rangle$ & $(1,1,1)$ & $(1,1,0)$ \\
\hline 3 & $\left\langle 1, \frac{\alpha(c-1)+c^{m}}{c \alpha}, \frac{\alpha-c^{m}}{c}\right\rangle$ & $\left(c^{m-1}, 0,1\right)$ & $\left(c^{m-1}, 1,0\right)$ \\
\hline$\vdots$ & $\vdots$ & $\vdots$ & $\vdots$ \\
\hline $4 t$ & $\left\langle 1, \alpha-c_{2}, \frac{c^{t}}{\alpha}\right\rangle$ & $\left(c_{2}, 1,0\right)$ & $\left(c^{t}-1,0,1\right)$ \\
\hline $4 t+1$ & $\left\langle 1, \frac{c^{t}-1}{\alpha}+\frac{c^{t}}{\alpha^{2}}, \frac{1}{\alpha}\right\rangle$ & $\left(c^{t}, 1,0\right)$ & $(0,0,1)$ \\
\hline $4 t+2$ & $\left\langle 1, \frac{\alpha-c^{m}}{\alpha\left(c^{l+1}-1\right)+c^{m}}, \frac{\alpha\left(\alpha-c^{m}\right)}{\alpha\left(c^{l+1}-1\right)+c^{m}}\right\rangle$ & $(1,1,0)$ & $(0,0,1)$ \\
\hline $4 t+3$ & $\left\langle 1, \frac{\alpha-c^{m}}{c^{l+1}}, \frac{\alpha\left(c^{c+1}-1\right)+c^{m}}{c^{l+1} \alpha}\right\rangle$ & $\left(c^{m-1-t}, 1,0\right)$ & $\left(c^{m-1-t}-1,0,1\right)$ \\
\hline$\vdots$ & $\vdots$ & $\vdots$ & $\vdots$ \\
\hline $4 m-4$ & $\left\langle 1, \alpha-c_{2}, \frac{c^{m-1}}{\alpha}\right\rangle$ & $\left(c_{2}, 1,0\right)$ & $\left(c^{m-1}-1,0,1\right)$ \\
\hline $4 m-3$ & $\left\langle 1, \frac{c^{m-1}-1}{\alpha}+\frac{c^{m-1}}{\alpha^{2}}, \frac{1}{\alpha}\right\rangle$ & $\left(c^{m-1}, 1,1\right)$ & $\left(c^{m-1}, 1,0\right)$ \\
\hline $4 m-2$ & $\left\langle 1, \frac{\alpha\left(c^{m}-1\right)+c^{m}}{c^{m} \alpha}, \frac{\alpha-c^{m}}{c^{m}}\right\rangle$ & $(1,0,1)$ & $(0,1,0)$ \\
\hline
\end{tabular}


In the table, we have written

$$
\phi_{0}=\alpha-c_{2} \quad \text { and } \quad \psi_{-1}=\frac{c^{m}}{\alpha} .
$$

As before, we deduce that

$$
\psi_{4 m-1}=\alpha\left(\frac{\alpha^{2}}{\alpha-c^{m}}\right)^{m}
$$

We have

$$
N\left(\psi_{4 m-1}\right)=1 \text { and } N\left(\psi_{i}\right) \neq 1 \quad \text { if } 0<i \leq 4 m-2 .
$$

Therefore, $\psi_{4 m-1}$ is the fundamental unit $\epsilon$ in $\mathscr{O}$ and the Voronoï-algorithm expansion period length is $l=4 m-1$.

4.2.3. The Jacobi-Perron algorithm. For this family the basis of the lattices $L_{k}, 0 \leq k \leq 4 m-2$, are not all given by the Jacobi-Perron algorithm expansion of $\left(\alpha\left(\alpha-c_{2}\right), \alpha\right)$. The transition matrices are given by

$$
\begin{gathered}
M_{0}=\left(\begin{array}{ccc}
c^{m}+c-1 & 1 & 0 \\
c^{m}-1 & 0 & 1 \\
1 & 0 & 0
\end{array}\right), M_{1}=\left(\begin{array}{ccc}
c^{m}+c-1 & 1 & 0 \\
0 & 0 & 1 \\
1 & 0 & 0
\end{array}\right), M_{2}=\left(\begin{array}{lll}
1 & 1 & 1 \\
1 & 1 & 0 \\
1 & 0 & 0
\end{array}\right), \\
M_{3}=\left(\begin{array}{ccc}
c^{m-1} & 0 & 1 \\
c^{m-1}-1 & 1 & 0 \\
1 & 0 & 0
\end{array}\right) ;
\end{gathered}
$$

for $1 \leq t \leq m-1$ :

$$
M_{4 t}=\left(\begin{array}{ccc}
c^{m}+c-1 & 1 & 0 \\
c^{t}-1 & 0 & 1 \\
1 & 0 & 0
\end{array}\right)
$$

for $1 \leq t \leq m-2$ :

$$
M_{4 t+1}=\left(\begin{array}{ccc}
c^{t} & 1 & 0 \\
0 & 0 & 1 \\
1 & 0 & 0
\end{array}\right), M_{4 t+2}=\left(\begin{array}{lll}
1 & 1 & 0 \\
0 & 0 & 1 \\
1 & 0 & 0
\end{array}\right), M_{4 t+3}=\left(\begin{array}{ccc}
c^{m-1-t} & 1 & 0 \\
c^{m-1-t} & 0 & 1 \\
1 & 0 & 0
\end{array}\right),
$$

and

$$
M_{4 m-3}=\left(\begin{array}{ccc}
c^{m-1} & 1 & 1 \\
c^{m-1} & 1 & 0 \\
1 & 0 & 0
\end{array}\right), M_{4 m-2}=\left(\begin{array}{lll}
1 & 0 & 1 \\
0 & 1 & 0 \\
1 & 0 & 0
\end{array}\right) .
$$

Let $a_{i}$ and $b_{i}$ be the integers defined by the Jacobi-Perron algorithm expansion, given by Levesque and Rhin [7], of $\left(\alpha\left(\alpha-c_{2}\right), \alpha\right)$, for which the period length is $\lambda=4 m+1$. For $0 \leq k \leq 4 m-4, k \neq 2$ and 3 , the transition matrices are given by the Jacobi-Perron algorithm :

if $k=0$ or $k=1$ :

$$
M_{k}=\left(\begin{array}{ccc}
a_{\lambda-k} & 1 & 0 \\
b_{\lambda-k} & 0 & 1 \\
1 & 0 & 0
\end{array}\right)
$$

if $4 \leq k \leq 4 m-4$ :

$$
M_{k}=\left(\begin{array}{ccc}
a_{\lambda-k-1} & 1 & 0 \\
b_{\lambda-k-1} & 0 & 1 \\
1 & 0 & 0
\end{array}\right)
$$


For $k=2$ and 3 we have the relation

$$
M_{3} M_{2}=\left(\begin{array}{ccc}
a_{\lambda-4} & 1 & 0 \\
b_{\lambda-4} & 0 & 1 \\
1 & 0 & 0
\end{array}\right)\left(\begin{array}{ccc}
a_{\lambda-3} & 1 & 0 \\
b_{\lambda-3} & 0 & 1 \\
1 & 0 & 0
\end{array}\right)\left(\begin{array}{ccc}
a_{\lambda-2} & 1 & 0 \\
b_{\lambda-2} & 0 & 1 \\
1 & 0 & 0
\end{array}\right),
$$

and for $k=4 m-3$ and $4 m-2$ we have the relation

$$
M_{4 m-2} M_{4 m-3}=\left(\begin{array}{ccc}
a_{1} & 1 & 0 \\
b_{1} & 0 & 1 \\
1 & 0 & 0
\end{array}\right)\left(\begin{array}{ccc}
a_{2} & 1 & 0 \\
b_{2} & 0 & 1 \\
1 & 0 & 0
\end{array}\right)\left(\begin{array}{ccc}
a_{3} & 1 & 0 \\
b_{3} & 0 & 1 \\
1 & 0 & 0
\end{array}\right) .
$$

Remark. For the quadratic form $F$, an isotropic vector in $L_{k}$ has the coordinates:

if $k=1$ or $k=2$ :

$$
\left(\begin{array}{c}
\alpha_{2}^{k-1} \\
1 \\
\alpha_{1}^{k-1}-b_{k-1}
\end{array}\right)
$$

if $k=3$ :

$$
\left(\begin{array}{c}
\alpha_{2}^{3} \\
\alpha_{1}^{3}-b_{3} \\
1
\end{array}\right)
$$

if $4 \leq k \leq 4 m-3$ :

$$
\left(\begin{array}{c}
\alpha_{2}^{k} \\
1 \\
\alpha_{1}^{k}-b_{k}
\end{array}\right)
$$

if $k=4 m-2$ :

$$
\left(\begin{array}{c}
\alpha_{2}^{k+1} \\
\alpha_{1}^{k+1}-b_{k+1} \\
1
\end{array}\right)
$$

where $\alpha_{2}^{i}$ and $\alpha_{1}^{i}$ are defined by the Jacobi-Perron algorithm expansion of $\left(\alpha\left(\alpha-c_{2}\right), \alpha\right)$.

\section{BIBLIOGRAPHY}

1. B.N. Delone and D.K. Faddeev, The theory of irrationalities of the third degree, Transl. Math. Monographs, vol. 10, Amer. Math. Soc., Providence, RI, 1964.

2. E. Dubois, Approximations diophantiennes simultanées de nombres algébriques. Calcul des meilleures approximations, Thèse de doctorat d'état, Univ. Pierre et Marie Curie, Paris, 1980.

3. E. Dubois and A. Fahrane, Unité fondamentale dans des familles d'ordres cubiques, Utilitas Math. 47 (1995), 97-115

4. A. Fahrane, Spécialisation de points extrémaux. Applications aux fractions continues et aux unités d'une famille de corps cubiques, Thèse, Univ. Caen, 1992.

5. F. Halter-Koch, Einige periodische Kettenbruchentwicklungen und Grundeinheiten quadratischer Ordnungen, Abh. Math. Sem. Univ. Hamburg 59 (1989), 157-169.

6. J. Kühner, On a family of generalized continued fraction expansions with period length going to infinity, J. Number Theory (to appear).

7. C. Levesque and G. Rhin, Two families of periodic Jacobi algorithms with period lengths going to infinity, J. Number Theory 37 (1991), 173-180. 
8. S. Louboutin, Minorations d'unités fondamentales. Applications, Nagoya Math. J. 130 (1993), $1-18$.

9. O. Perron, Grundlagen für eine Theorie des Jacobischen Kettenbruchalgorithmus, Math. Ann. 64 (1907), 1-76.

10. H. J. Stender, Eine Formel für Grundeinheiten in reinen algebraischen Zahlkörpern dritten, vierten und sechsten Grades, J. Number Theory 7 (1975), 235-250.

11. G. F. Voronoi, On a generalization of the algorithm of continued fractions, Doctoral Dissertation, Warsaw, 1896 (in Russian).

12. H. C Williams, The period length of Voronoi's algorithm for certain cubic orders, Publ. Math. Debrecen 37 (1990), 245-265.

URA CNRS 399, Département de MATHÉmatiques et INFORMATiQue, UFR MIM, UNiVERSité de MeTZ, Ile du SAulcy, 57045 Metz Cedex 01, France

E-mail address: adam@poncelet.univ-metz.fr 